\title{
Artificial grammar learning in Alzheimer's disease
}

\author{
PAUL J. REBER, LUCY A. MARTINEZ, and SANDRA WEINTRAUB \\ Northwestern University, Evanston, Illinois
}

\begin{abstract}
Patients with early Alzheimer's disease (AD) exhibit impaired declarative memory although some forms of nondeclarative memory are intact. Performance on perceptual nondeclarative memory tasks is often preserved in $\mathrm{AD}$, whereas conceptual nondeclarative memory is often impaired. A conceptual nondeclarative learning task that has been studied in amnesic patients is the artificial grammar learning (AGL) task. Healthy participants and patients with impaired declarative memory both acquire information about an underlying rule structure in this task and exhibit the ability to identify rule-conforming items, despite the subjective experience of guessing at the response. In this study, 12 patients diagnosed with early $\mathrm{AD}$ were tested on the AGL task and a matched recognition task. The patients were able to reliably distinguish rule-conforming items from others, indicating successful AGL. Performance of the $\mathrm{AD}$ patients was impaired, relative to controls, on a similar recognition task, although they were found to use information about the grammaticality of study items in an attempt to improve their recognition performance. The $\mathrm{AD}$ patients showed a dissociation similar to that seen in anterograde amnesia: impaired recognition memory in conjunction with successful AGL. This finding suggests that the brain areas that support AGL are not compromised early in the course of $\mathrm{AD}$. In addition, the nondeclarative memory of the $\mathrm{AD}$ patients acquired during $\mathrm{AGL}$ appeared to influence their performance on a declarative memory task, suggesting an interaction between this nondeclarative memory task and declarative memory.
\end{abstract}

Alzheimer's disease (AD) is characterized by an early and progressive impairment in memory function. Early in the course of $\mathrm{AD}$, damage occurs to the medial temporal lobe (MTL), leading to impairment in declarative memory, the ability to learn new facts and events. The neuropathology of $\mathrm{AD}$ becomes progressively widespread, involving particularly the association areas of the temporal, parietal, and frontal lobes (Braak \& Braak, 1991; Delacourte et al., 1999; Hyman, Van Horsen, Damasio, \& Barnes, 1984; Kemper, 1984). The study of preserved memory function in patients with anterograde amnesia resulting from acute damage to the MTL has shown that there are a number of types of memory that are intact when declarative memory is impaired (Squire, Knowlton, \& Musen, 1993). These phenomena are collectively termed nondeclarative memory. Specific nondeclarative memory tasks have been shown to depend on different areas of the brain outside the MTL, including the basal ganglia, the cerebellum, and sensory cortical areas. The study of nondeclarative memory in AD patients is complicated by the fact that neuropathology extends beyond the MTL and may affect some of the areas that support some types of nondeclarative memory.

This research was supported by a grant from the Illinois Department of Public Health, Alzheimer's Disease Research Fund (to P.J.R.), and by a pilot project award from the Northwestern Alzheimer's Disease Center (AG13854). The authors thank Michael Levitt, Anjali Raja, Chris Fahey, and Grace Chong for assistance in data collection and Nancy Johnson for assistance in patient recruitment. Correspondence concerning this article should be addressed to P. J. Reber, Department of Psychology, Northwestern University, 2029 Sheridan Road, Evanston, IL 60201 (e-mail: preber@northwestern.edu).
A number of studies have examined the phenomenon of priming in patients with early AD (see Fleischman \& Gabrieli, 1998, for a review). In most perceptual priming studies, performance is intact in AD patients, likely reflecting the sparing of occipital and occipito-temporal cortical areas early on (e.g., Keane, Gabrieli, Fennema, Growdon, \& Corkin, 1991). In contrast, conceptual priming does not appear to be intact in AD patients (e.g., Monti et al., 1996), which may be due to the fact that the pathological changes occur in areas that also support semantic memory (reviewed in Nebes, 1989, 1992). As Fleischman and Gabrieli (1998) have noted, there are exceptions to this general distinction between intact perceptual and impaired conceptual priming in AD. There are perceptual priming tasks, such as degraded picture naming, that have been reported to be impaired in AD (Corkin, 1982; Heindel, Salmon, \& Butters, 1990). In addition Gabrieli et al. (1999) have reported intact conceptual priming on category exemplar verification, although category exemplar generation was impaired in the same study, suggesting that the need to generate a response may play a role in predicting what types of priming tasks are impaired in $\mathrm{AD}$. The challenge is to interpret the pattern of impaired and intact memory in the context of the neuropathology of early AD. Even in relatively early $\mathrm{AD}$, when dementia is "mild," neurofibrillary tangles are observed throughout the temporal cortex, the anterior prefrontal cortex, and the inferior parietal cortex (Delacourte et al., 1999). The widespread neuropathology suggests that the conceptual or generative nondeclarative memory tasks on which even mild AD patients are impaired may depend more heavily on the fronto-temporal 
cortical areas that may be partly damaged in early AD. Intact posterior cortical areas may support the types of perceptual priming that are intact in AD. Consistent with the behavioral pattern, neuroimaging studies provide additional evidence that posterior cortical areas support perceptual priming (Schacter \& Buckner, 1998), whereas the prefrontal cortex appears to be more associated with conceptual priming (e.g., Wagner, Koutstaal, Maril, Schacter, \& Buckner, 2000).

The impairment of AD patients on some conceptual nondeclarative memory tasks supports the idea that there are dissociations within nondeclarative memory for different types of tasks. The conceptual tasks on which AD patients are impaired are performed at a normal rate by amnesic patients with selective damage to the MTL, indicating that the impairment in the $\mathrm{AD}$ patients is not based on their MTL dysfunction. The pattern of intact and impaired nondeclarative memory in AD simultaneously provides information about dissociations between nondeclarative memory phenomena and about what memory functions are intact in $\mathrm{AD}$. This dissociation between $\mathrm{AD}$ and pure amnesia suggests that the brain areas that support these conceptual tasks are intact in amnesic patients but impaired in AD patients. In contrast, the brain areas that support most perceptual priming tasks appear to be intact in both groups.

Comparison of the neurological profiles of groups that exhibit a dissociation within nondeclarative memory has been used in other patient groups to provide additional information about the neural substrates of specific nondeclarative memory tasks. An example of this is evident in the dissociation between amnesic patients and patients with Parkinson's disease (PD) on some perceptual-motor and habit-learning tasks. Perceptual-motor sequence learning is intact in amnesic patients (Nissen \& Bullemer, 1987; P. J. Reber \& Squire, 1994) but is impaired in PD patients (Jackson, Jackson, Harrison, Henderson, \& Kennard, 1995), suggesting that the pathological involvement of the basal ganglia in PD is associated with impaired learning on this nondeclarative memory task. Consistent with this idea, patients with AD demonstrate intact perceptualmotor sequence learning (Knopman \& Nissen, 1987), as well as rotary pursuit (Eslinger \& Damasio, 1986; Heindel, Salmon, Shults, Walicke, \& Butters, 1989), reflecting the normal function of the basal ganglia in early AD. In contrast to this dissociation, both PD patients and amnesic patients exhibit normal learning of artificial grammars (Knowlton, Ramus, \& Squire, 1992; P. J. Reber \& Squire, 1999a). Thus, the dissociation within forms of nondeclarative memory demonstrates that there are multiple brain areas supporting memory on different nondeclarativememory tasks.

In addition to perceptual and motor-oriented tasks, such as priming and sequence learning, nondeclarative memory has been demonstrated in more complex cognitive tasks, such as visual category learning (Knowlton \& Squire, 1994) and learning rule structures in an artificial grammar (Knowlton et al., 1992; Knowlton \& Squire, 1994,
1996). These tasks have extended the domain of nondeclarative memory into complex, high-level cognition. For many of these tasks, the neural substrate of the supporting nondeclarative memory is not well understood. For example, one form of nondeclarative category learning (probabilistic classification) appears to be impaired in PD patients (Knowlton, Mangels, \& Squire, 1996), suggesting that the basal ganglia play a role in learning for that task. However, PD patients show normal learning of a visual category based on an underlying prototype (P. J. Reber \& Squire, 1999a), indicating that the basal ganglia are not critical for this nondeclarative category-learning task. Even within tasks of nondeclarative category learning, there may be different types of nondeclarative memory involved.

Studies of AD patients have just begun to examine these higher level cognitive nondeclarative memory tasks. Kéri and colleagues examined the performance of early AD patients on a visual category-learning task (Kéri, Kálmán, Kelemen, Benedek, \& Janka, 2001; Kéri et al., 1999). This task has been reported to be learned normally by amnesic patients (Knowlton \& Squire, 1994) and PD patients (Peigneux, Meulemans, Van Der Linden, Salmon, \& Petti, 1999; P. J. Reber \& Squire, 1999a). Impaired learning was observed in patients with moderately advanced $\mathrm{AD}$, whereas patients with mild $\mathrm{AD}$ exhibited normal learning. On the basis of functional neuroimaging studies, intact learning on this task is thought to be supported by cortical changes in visual-processing areas (P. J. Reber, Stark, \& Squire, 1998a, 1998b). The report of Kéri et al. (2001) suggests that the progression of neuropathology from mild to moderate $\mathrm{AD}$ affects the brain areas that support this type of nondeclarative memory.

The previous reports of intact nondeclarative memory in AD for priming (Keane et al., 1991) and visual category learning (Kéri et al., 2001) suggest an intact nondeclarative memory system for perceptually oriented tasks in AD. However, the complexity of the artificial grammar learning (AGL) task suggests that a representation that is at least somewhat more conceptual or abstract is required. In the AGL task, participants are shown a set of letter strings consisting of consonants (rendering them unpronounceable; e.g., PVPXTJ), that conform to an underlying set of rules. The rules are typically depicted graphically (Figure 1) as a state transition diagram. The rules indicate the order in which the letters can occur. Strings that follow the rules are described as being grammatical. Nongrammatical strings are constructed from the same letters, but the order of the strings violates the rules in one or two places. The study phase of an AGL experiment consists of exposure to grammatical letter strings without any information being provided about the grammatical nature of the strings. Instead, participants are asked to reproduce, memorize, or observe the strings. After completing the study phase, the participants are surprised with the information that the strings conform to an underlying rule structure, and although they typically indicate that they were unaware of these rules, they exhibit knowledge of the rule structure by being able to indicate the grammaticality of novel strings 

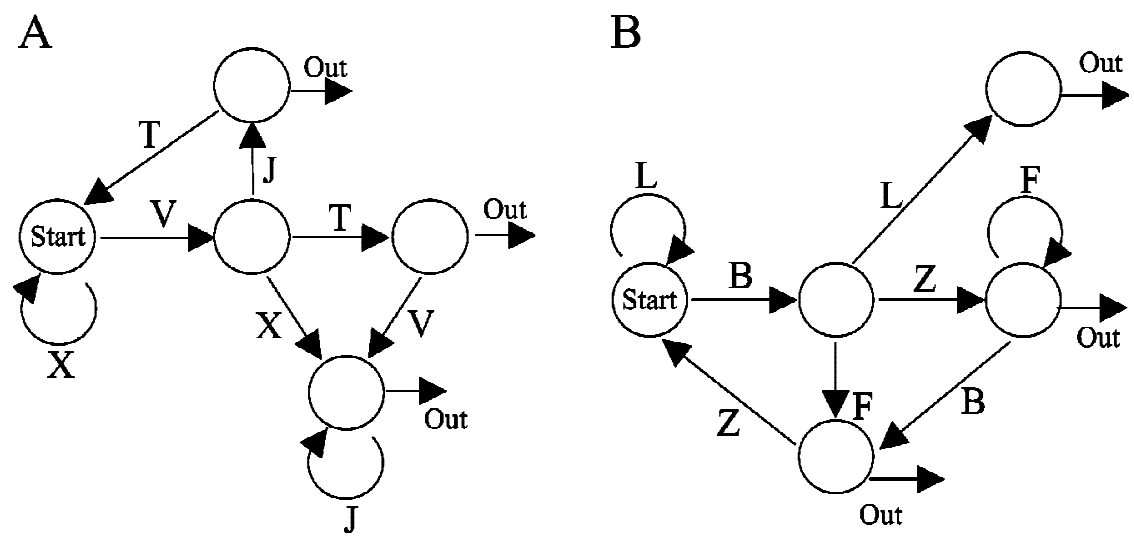

Figure 1. Graphical representation of the artificial grammar rule systems. To generate a grammatical string, one begins with the start state and chooses one of the two possible transitions (arrows), recording the letter associated with the transition and moving to the indicated state. One continues to choose transitions, recording letters and changing state until an "End" transition is selected. Some transitions are loops, in that they maintain the current state (but the letter is still recorded). The recorded string conforms to the rules of the grammar. A large number of grammatical strings can be defined on the basis of the particular transitions chosen at each state. (A) Grammar A. Examples of grammatical strings used during study (VTVJ, XXXVT, VJTXVJ) and at test (XVTVJ, XVX, VTVJJ). Nongrammatical strings were derived from grammatical strings, with a rule violation added (underlined), (e.g., IJTVJ, V $\underline{\mathrm{xVJ}}$, XVTXVJ). (B) Grammar B. Examples of grammatical strings used during study (LBZFF, LLLBL, BFZBZF) and at test (LBFZBL, LBL, BZBZBL). Nongrammatical strings were derived from grammatical strings (as in A) but contained a rule violation (e.g., LZLBB, BLFZ, BZELLL).

at a level better than chance (A. S. Reber, 1967, 1989). Grammatical and nongrammatical strings look very similar on the surface, and the ability to discriminate between them implies some knowledge of the underlying rules.

For some category-learning tasks, concerns have recently been raised about the possibility of participants' achieving successful performance on the basis of extracting the structure of the stimuli during the test (Palmeri \& Flannery, 1999). In this case, performance could reflect the operation of short-term memory for recent stimuli, rather than long-term nondeclarative memory. For AGL, this issue was addressed in P. J. Reber \& Squire (1999a) by having a group of control participants attempt to perform the AGL task without having been previously exposed to the study items. Performance was at chance levels, reflecting the fact that it is difficult to perceive the structure of AGL test items well enough to acquire the underlying knowledge during the test.

The degree of abstract representation required in AGL has been the subject of considerable debate. It has been pointed out that the underlying rules of an artificial grammar can be represented as a set of conditional probabilities that reflect frequently recurring fragments (or chunks) of letter strings (Perruchet, 1994; see also Vokey \& Brooks, 1992). However, participants have also been shown to be able to make grammaticality judgments in a letter-set transfer version of the AGL, in which the letters used at study and at test are changed (A. S. Reber, 1989). Changing the letters used in the strings changes the surface features of the strings considerably and suggests that the rep- resentation of the grammatical rules has at least an element of abstract (with respect to the surface characteristics) representation.

In a series of studies of amnesic patients, AGL was found to be intact even when declarative memory for letter strings was severely impaired (Knowlton et al., 1992; Knowlton \& Squire, 1994, 1996). The intact AGL in amnesic patients also extended to the letter-set transfer version of the task (Knowlton \& Squire, 1994), indicating the existence of an abstract, conceptual form of nondeclarative memory. Knowlton and Squire (1996) also attempted to separate the contributions of fragment-based grammar knowledge from a more abstract representation and found evidence that performance was influenced by both elements.

For the purposes of the present study, the key point is that, whether AGL is supported principally by fragments or by underlying rule abstraction, this ability reflects a more complex and conceptual form of nondeclarative memory than is required to support most forms of perceptual priming. Even identification of a recombination of letter string fragments suggests a more cognitively complex process than simply decreasing response time to the re-presentation of a previously seen stimulus (as in perceptual identification priming). Examining the performance of $\mathrm{AD}$ patients on the AGL task indicates whether the brain areas that support this type of learning are affected by the neuropathology of early AD. The AGL task may have an element of perceptual learning involved in it, but success by the AD patients on the AGL task would indicate that this relatively 
conceptual nondeclarative memory task is preserved early in the course of $\mathrm{AD}$.

\section{METHOD}

\section{Participants}

Twelve patients (6 men and 6 women) with a diagnosis of probable AD (McKhann et al., 1984) were tested. Only patients with mild dementia, as indicated by a clinical dementia rating of 1 (Morris, 1993), were studied. The patients averaged 76.8 years in age (range, 67-87) and had completed an average of 14.5 years of education (range, 11-20). Twelve healthy controls (4 men and 8 women) were matched to the $\mathrm{AD}$ patients in mean age and education (mean age, 75.1 years; range, 68-92; mean years of education, 16.4; range, 12-20). All the patients were identified as having impaired declarative memory, on the basis of performance on the word list learning task from the Consortium to Establish a Registry for Alzheimer's Disease (CERAD) battery (Morris et al., 1989) and the Logical Memory II and Visual Reproduction II subscales (delayed recall) of the Wechsler Memory Scale-Revised (Weschler, 1987; see Table 1).

Although all $\mathrm{AD}$ patients exhibit significant impairments in memory function, the diagnosis of $\mathrm{AD}$ requires impairment in at least one other cognitive domain. In Table 2, patients' performance is shown for the Mini Mental State Examination (MMSE; Folstein, Folstein, $\&$ McHugh, 1975), a test of global dementia severity, for the Category Fluency Test (Morris et al., 1989), a test of persistence and semantic memory and language, for the Constructions subtest of the CERAD battery (Morris et al., 1989), which assesses visual spatial ability and executive functions, for the Visual Target Cancellation Test (Rosler et al., 2000), which assesses visual attention, and for the Boston Naming Test (Kaplan, Goodglass, \& Weintraub, 1983). Each test has specific age- and/or education-normed scores. Individual $Z$ scores reflecting the patient's performance relative to age and education norms are reported.

It is apparent that the AD patients performed deficiently on one or more of these tests, with scores falling more than one standard deviation below age-appropriate means in many cases (Table 2). On the MMSE, three patients had scores in the very mildly impaired range (Patients 5, 7, and 11), eight had scores in the range of mild impairment (Patients 1, 3, 4, 6, 8, 9, 10, and 12), and one had a score in the moderate impairment range (Patient 2). All eight of the patients who received the Category Fluency Test scored more than one standard deviation below the mean. The impairments on the other tests varied from patient to patient. In general, each of the AD patients had additional cognitive deficits outside of their general memory impairment.

\section{Materials}

Twenty grammatical strings were constructed from the artificial grammars shown in Figure 1, to be used in the study (reproduction) phase. An additional 20 grammatical strings were constructed for the test, along with 20 strings that each contained a violation of the grammar rules. The strings were each printed with a 72-point font and pasted onto individual $3 \times 5$ in. index cards for display.

\section{Procedure}

Three tasks were administered over two sessions at least 1 week apart. In the first session, the AGL task was administered twice, with a short break between tests. Each administration of the AGL task contained a study phase and a test phase (see below), so the structure of this session was a study phase, a grammaticality judgment test, a break, a study phase, and a second grammaticality judgment test. Different sets of grammatical stimuli were used for the two tests, and the order of presentation of the two was counterbalanced across participants. In a second session, a recognition task was administered that followed a similar structure: a study phase followed by a recognition memory test.

Study phase. In all of the study phases, the participants were shown letter strings 3-6 letters in length one at a time for $9 \mathrm{sec}$. After the item had been viewed, it was removed from view, and the participants attempted to reproduce the item from memory with pencil and paper. If the item was reproduced incorrectly, two more chances were given, after which it was copied (if necessary). A set of 20 study strings was presented this way and then were presented a second time in a new order (a total of 40 study trials). After the study phase, there was a 5-min conversation-filled break, followed by the test.

Table 1

Demographic Information and Memory and Global Cognitive Test Scores of the Alzheimer's Disease (AD) Patients and Controls

\begin{tabular}{|c|c|c|c|c|c|c|c|c|c|}
\hline Patient & Age & Education & $\begin{array}{c}\text { CERAD } \\
\text { Word List } \\
\text { Trials 1-3 }\end{array}$ & $\begin{array}{c}\text { CERAD } \\
\text { Delayed } \\
\text { Recall } \\
\end{array}$ & $\begin{array}{c}\text { CERAD } \\
\text { Recognition } \\
\text { Hits } \\
\end{array}$ & FA & $\begin{array}{c}\text { Logical } \\
\text { Memory II }\end{array}$ & $\begin{array}{c}\text { Visual } \\
\text { Reproduction II }\end{array}$ & MMSE \\
\hline 1 & 87 & 16 & 17 & 0 & 6 & 3 & 0 & 0 & 22 \\
\hline 2 & 77 & 18 & 11 & 0 & 8 & 7 & 0 & 0 & 19 \\
\hline 3 & 67 & 12 & 11 & 3 & 10 & 0 & 1 & 0 & 23 \\
\hline 4 & 84 & 12 & 13 & 0 & 9 & 1 & 0 & 0 & 23 \\
\hline 5 & 72 & 16 & 11 & 0 & 7 & 5 & 0 & 0 & 28 \\
\hline 6 & 76 & 14 & 8 & 0 & 7 & 3 & 0 & 0 & 23 \\
\hline 7 & 83 & 13 & 19 & 2 & 10 & 0 & 6 & 7 & 26 \\
\hline 8 & 86 & 20 & 14 & 1 & 6 & 0 & 0 & 5 & 20 \\
\hline 9 & 76 & 12 & 16 & 1 & 8 & 6 & NA & NA & 25 \\
\hline 10 & 74 & 12 & 14 & 2 & 8 & 1 & 2 & NA & 22 \\
\hline 11 & 73 & 11 & 15 & 3 & 10 & 1 & 8 & 10 & 28 \\
\hline 12 & 66 & 18 & 8 & 2 & 8 & 1 & 8 & 0 & 22 \\
\hline Average for $\mathrm{AD}$ & 76.8 & 14.5 & 13.1 & 1.2 & 8.1 & 2.3 & 2.3 & 2.2 & 23.4 \\
\hline$S D$ & 7.0 & 3.0 & 3.4 & 1.2 & 1.4 & 2.5 & 3.3 & 3.7 & 2.8 \\
\hline Average for controls & 75.1 & 16.4 & 25.3 & 8.7 & 9.9 & 0 & 28.4 & 35.1 & 29.2 \\
\hline$S D$ & 7.2 & 2.8 & 2.6 & 1.6 & 0.3 & 0.0 & 10.2 & 4.2 & 1.0 \\
\hline
\end{tabular}

Note-Individual scores are shown for the 12 AD patients for the CERAD Word List test (maximum = 30; Morris et al., 1989), CERAD delayed recall (maximum = 10; Morris et al., 1989), CERAD recognition (FA $=$ false alarms), the Logical Memory II and Visual Reproduction II subscales (delayed recall scores) of the WMS-R (Weschler, 1987; maximum $=50$ and 41, respectively), and the MMSE (Folstein, Folstein, \& McHugh, 1975). Means for the 12 control participants are shown for comparison. 
Table 2

Additional Neuropsychological Test Scores for the Alzheimer's Disease (AD) Patients and Control

\begin{tabular}{|c|c|c|c|c|c|c|c|c|}
\hline Patient & $\begin{array}{c}\text { Category } \\
\text { Fluency Test }\end{array}$ & $z$ & $\begin{array}{c}\text { Constructions } \\
\text { Test }\end{array}$ & $z$ & $\begin{array}{c}\text { Target Cancellation } \\
\text { Errors }\end{array}$ & $z$ & $\begin{array}{c}\text { Boston } \\
\text { Naming Test }\end{array}$ & $z$ \\
\hline 1 & 11 & -1.48 & 10 & -0.30 & 0 & -0.50 & 43 & -1.22 \\
\hline 2 & 11 & -1.48 & 9 & -1.30 & 0 & -0.50 & 58 & 1.02 \\
\hline 3 & 10 & -1.71 & 10 & -0.30 & 2 & 1.17 & 41 & -3.40 \\
\hline 4 & 6 & -2.67 & 10 & 0.00 & 7 & 5.33 & 51 & -0.08 \\
\hline 5 & 3 & -2.66 & 4 & -4.62 & 18 & 14.50 & 41 & -2.60 \\
\hline 6 & 9 & -2.07 & 5 & -3.85 & 11 & 8.67 & 40 & -1.89 \\
\hline 7 & 10 & -1.84 & 11 & 0.70 & 3 & 2.00 & 57 & 0.78 \\
\hline 8 & 11 & -1.62 & 9 & -1.30 & OT & NA & 51 & -0.08 \\
\hline 9 & NA & NA & 9 & -0.77 & OT & NA & 50 & -0.27 \\
\hline 10 & NA & NA & 5 & -5.30 & OT & NA & 27 & -5.31 \\
\hline 11 & NA & NA & 10 & 0.63 & 6 & 4.50 & 56 & 0.30 \\
\hline 12 & NA & NA & 11 & 0.78 & 2 & 1.17 & 19 & -8.53 \\
\hline Average for AD & 8.88 & -1.94 & 8.6 & -1.30 & 5.4 & 4.04 & 44.5 & -1.77 \\
\hline$S D$ & 2.90 & 0.49 & 2.50 & 2.12 & 5.90 & 4.93 & 12.02 & 2.84 \\
\hline Average for controls & 24.46 & 1.46 & 10.58 & 0.37 & 0.46 & -0.12 & 58.13 & 0.84 \\
\hline$S D$ & 5.1 & 1.04 & 0.67 & 0.56 & 0.89 & 0.74 & 2.05 & 0.41 \\
\hline
\end{tabular}

Note-Individual scores are shown for the 12 AD patients on the Category Fluency Test (Morris et al., 1989), CERAD Constructions subtest (maximum $=11$, Morris et al., 1989), Target Cancellation (best score $=0$ errors; OT $=$ unable to complete or did not take the test), and the Boston Naming Test (maximum =60; Kaplan, Goodglass, \& Weintraub, 1983). Norms were also used to calculate $z$ scores (negative numbers reflect increasing impairment for all tests except the Target Cancellation Test, where positive $z$ scores reflect an increasing number of errors). Means for the 12 control participants are shown for comparison.

Grammaticality test. For this test, after the break, the participants were told the following:

\begin{abstract}
All the items you saw before the break were created from a complex set of rules. Now I'm going to show you new items. For each item I want you to decide if you think it was made from the same set of rules. If you think it was made from the same set of rules say "yes;" if you think the item was not made from the set of rules say "no." The rules are very complex, so you may not be able to figure them out. You may want to go on a "gut feeling" as to whether the item follows the rules. Make your response even if you have to guess.
\end{abstract}

The participants were then shown 40 novel letter strings, 20 of which accurately followed the rules of the grammar and 20 of which violated those rules in one or two places. The average length of the strings in the study phase was 4.9 letters (range, 3-6). Test items averaged 5.0 letters in length (range, 3-6; mean length for the grammatical strings was 5.2 letters; the nongrammatical strings averaged 4.8 letters).

Recognition task. For the recognition test (given on a separate session from the AGL tests), the same stimuli were used as those that had been seen in an AGL task from the previous week (half the participants saw stimuli from Grammar A, and half from Grammar B). In the recognition task, the participants were given another study (reproduction) phase that was exactly the same as that used in the AGL test. After the study phase, the participants were shown 40 strings, 20 old and 20 new, 1 at a time, and were asked to say "yes" if they believed the string was one that they had seen during the study (reproduce) phase and "no" if they thought that the string was new. The study items used for the recognition test were the identical items that had been used for one of the grammars a week earlier. Thus, all the old items during the recognition test were also grammatical. Half of the new strings (foils) were novel grammatical items, and half were novel nongrammatical items. This design permitted evaluation of the effect of the grammaticality of the stimulus items on a conscious, recognition memory judgment.

\section{RESULTS}

\section{Study Phase Performance}

The study phase required reproducing each of the 20 study items, twice each, from short-term memory (the minimum number of trials to complete this phase was 40). The patients made an average of 11.1 errors (range, $0-45$ ) while reproducing the item from memory during each study phase (over the 40 trials). The controls made an average of 3.4 errors (range, $0-14$ ), and the difference between the groups was marginal $[t(22)=1.87, p<.10]$. The poorer average performance of the AD patients was due largely to 2 patients who averaged 32.5 and 41 errors across the two study phases (the largest number of study trial errors for a control was 14; the next largest number of study trial errors for an AD patient was 10).

\section{AGL Grammaticality Performance}

Performance on the artificial grammar test is shown in Figure 2, panel A, for the patients and the controls. The AD patients were correct on an average of $57.6 \%( \pm 1.8$ $S E$ ) of the trials when discriminating between grammatical and nongrammatical items. The controls were correct on an average of $63.8 \%( \pm 2.6 S E)$ of the trials. Both groups performed better than chance $[50 \% ; t(11)=4.28$, $p<.01$, for the $\mathrm{AD}$ patients, and $t(11)=5.12, p<.001$, for the controls]. The difference in performance between the two groups did not reach statistical significance $[t(22)=1.77, p<.10]$.

Performance was similar for both grammaticality sessions for both groups. The AD patients scored $58.1 \%$ correct $( \pm 2.0)$ on the first test and $57.1 \%$ correct $( \pm 2.1)$ on the second $[t(11)=0.50]$. The controls scored $61.9 \%$ $( \pm 2.8)$ and $64.3 \%( \pm 2.5)$ correct on the first and the second tests, respectively $[t(11)=1.21]$. A $2 \times 2$ analysis of variance (ANOVA) comparing the two groups across both tests showed a marginal effect of group $[F(1,22)=3.13$, $p<.10]$, no effect of test $(F<1)$, and no interaction $[F(1,22)=1.91, p>.15]$.

No overall relationship between number of study trials and grammaticality judgment performance was observed 

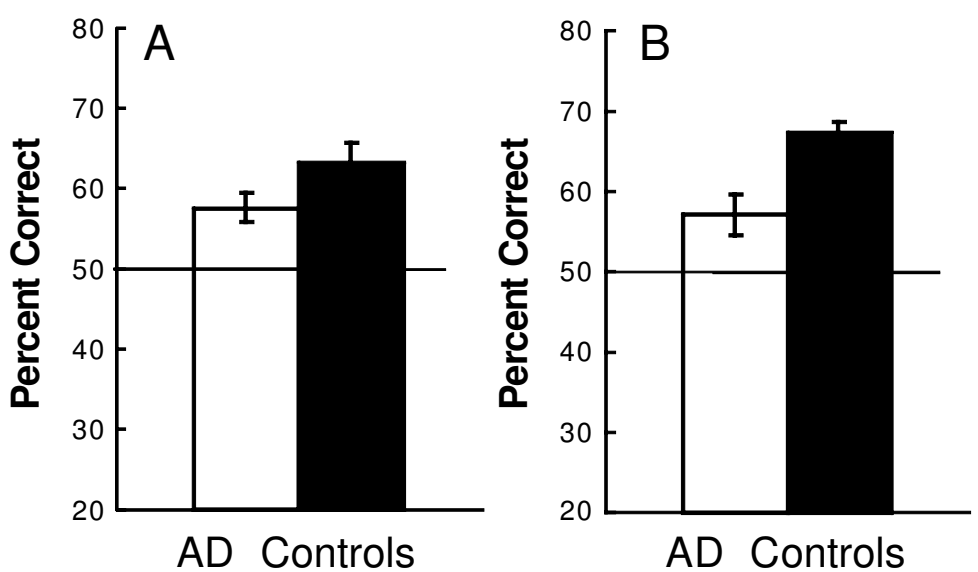

Figure 2. (A) Performance on the artificial grammar task. Percent correct indicates the degree to which the Alzheimer's disease (AD) patients and the controls were able to discriminate between novel grammatical items and nongrammatical items on the grammaticality judgment task. Both groups performed significantly better than chance $(t \mathrm{~s}>4.2, p \mathrm{~s}<.01)$. (B) Performance on the recognition test for letter strings. Percent correct indicates the ability of the AD patients and controls to identify strings that had been previously presented during the study phase. The controls performed significantly better than the AD patients $[t(22)=3.51, p<.01]$, although both groups performed better than chance $[t \mathrm{~s}>2.7, p \mathrm{~s}<.05]$.

( $r=-.04$ for both groups combined; $r=.12$ within the $\mathrm{AD}$ patient group).

\section{Recognition Performance}

Overall performance on the recognition test is shown in Figure 2, panel B. The AD patients were correct on an average of $57.1 \%( \pm 2.6 S E)$ of the trials when discriminating between previously seen and novel letters. The controls were correct on an average of $67.3 \%( \pm 1.4)$ of the trials. Both groups performed better than chance $[50 \%$; $t(11)=2.77, p<.05$, for the AD patients, and $t(11)=$ $12.4, p<.001$, for the controls]. The performance of the controls was significantly better than the performance of the AD patients $[t(22)=3.51, p<.01]$.

Because the study items for the recognition test were all grammatical items, it is possible that the participants could have used the grammaticality of the recognition test items to improve performance on the recognition test. To assess this, the endorsement rates for old grammatical items, new grammatical items, and new nongrammatical items were compared (Figure 3 ). A $3 \times 2$ ANOVA of item type (within subjects) and group indicated that there was an overall (linear) effect of item type $[F(1,22)=93.3, p<.001]$, and a significant group $\times$ trial type interaction reflected the significant differences in old/new and grammatical/ nongrammatical factors for the patients and the controls $[F(1,22)=12.7, p<.01]$. This analysis raised the possibility that the AD patients had used grammaticality information to support recognition judgments in the absence of declarative memory. In this case, it would be expected that the memory-impaired patients would exhibit a smaller difference in endorsement rates for old and new grammati- cal items on the recognition test. A $2 \times 2$ ANOVA of item type and group indicated that there was a main effect of item type $[F(1,22)=9.78, p<.01]$ and a marginal interaction between item type and group $[F(1,22)=3.52$, $p<.08]$, with no reliable effect of group $[F(1,22)=$ 0.57]. However, 4 of the AD patients performed well on the recognition test (65\%), suggesting that they may have had some residual declarative memory contributing to their recognition performance. Since our hypothesis was that grammaticality information would influence recognition when declarative memory was impaired, we reexamined endorsement rates for old and new grammatical items, comparing the 8 poorer performing patients with the controls, and found a reliable interaction between item type and group $[F(1,18)=5.45, p<.05]$, as well as a main effect of item type $[F(1,18)=4.69, p<.05]$ and no effect of group $[F(1,19)=1.17, p>.25]$. Thus, the memorydisordered patients appear to have been strongly influenced by grammaticality during recognition (improving their overall performance, since the targets were grammatical). Reflecting this, if the recognition responses had been scored as if the test were a grammaticality test (i.e., scoring a "yes" response as correct for old and new grammatical strings), the AD patients would have obtained a score of $66.7 \%$ correct, similar to their performance on the earlier grammaticality tests.

\section{DISCUSSION}

Patients with relatively mild AD exhibited implicit learning of the rules of an artificial grammar. Both patients and nondemented controls acquired knowledge of the gram- 


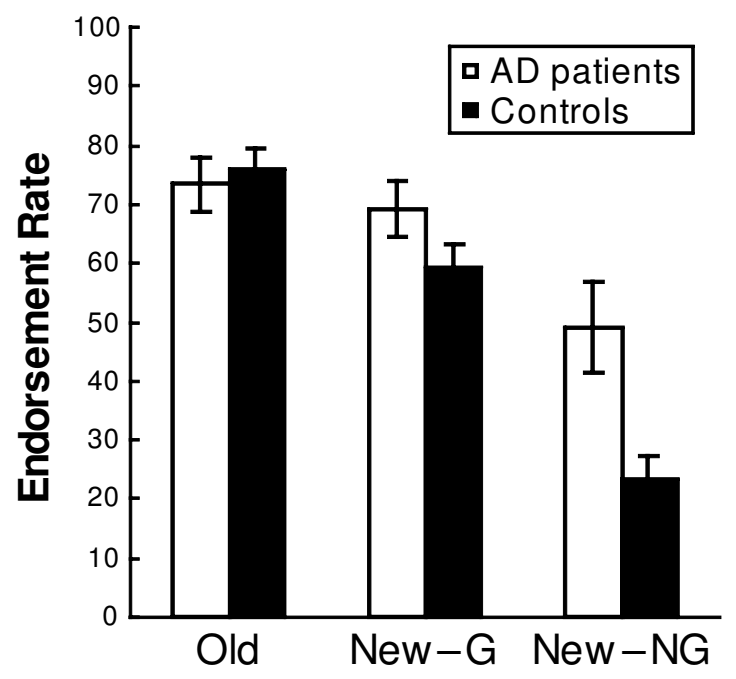

Figure 3. Performance on the recognition test as a function of the grammaticality of the test items. All "Old" items were grammatical and had been shown during the study phase. The "New" items contained equal numbers of grammatical (New-G) and nongrammatical (New-NG) letter strings. Both controls and Alzheimer's disease (AD) patients made false alarms to New-G items (incorrectly responding "yes" if the item had been seen previously) at a higher rate than to the New-NG items $(t \mathrm{~s}>\mathbf{2 . 8}$, $p<.05)$. The controls also discriminated between the Old and the New-G items effectively $[t(11)=3.90, p<.01]$, reflecting their recognition of the studied items. The AD patients did not discriminate between Old and New-G letter strings, reflecting their impaired recognition memory for the letter strings and suggesting a reliance on grammaticality during the recognition test.

mar after incidental study of a set of letter strings that conformed to those rules. The learning process occurred incidentally, and although the participants were not necessarily aware of learning, they were subsequently successful at discriminating between grammatical and nongrammatical letter strings. When recognition memory was tested following an identical study phase, the AD patients were impaired relative to the controls. These results are consistent with previous reports dissociating AGL and recognition memory (e.g., Knowlton et al., 1992) and extend this finding to $\mathrm{AD}$ patients.

The overall level of learning exhibited by the AD patients was marginally inferior to the controls' performance. This trend may suggest that the progression of AD eventually affects brain regions that support AGL. Another possibility is that some of the patients may have had difficulty understanding the test instructions, which were moderately complex. All AD patients exhibit mild levels of dementia, and some patients might have become confused by the grammaticality instructions that referred to rules that they did not consciously know (see the section on AGL test methods, above). If this led to adoption of a maladaptive strategy for responding on the test, some patients may have done poorly even though they had learned the grammar. In comparison, on the recognition task, which does not refer to grammaticality, the AD patients' responses were clearly influenced by the grammaticality, and they would have been scored $66.7 \%$ correct if this task had been scored as a grammaticality test (rather than as a recognition test). Although the AD patients did exhibit significant learning on the AGL task, implicit learning tasks with simpler instructions and demand characteristics may be more effective for assessing intact learning.

An additional way in which the controls might have had an advantage over the AD patients is that on the second AGL test, they may have realized the nature of the test and could have used intact declarative memory to support learning. However, the controls did not perform significantly better overall on the second test $(62 \%$ correct on the first test, $64 \%$ correct on the second), suggesting that this additional information was not helpful to them.

Despite all the reasons the patients might have performed poorly on AGL, the AD patients exhibited significant learning of the rules of the artificial grammar. This suggests that the most commonly implicated areas in the neuropathology of $\mathrm{AD}$ are unlikely to be critically involved in supporting AGL. This result is consistent with previous reports of intact AGL in amnesic patients who had damage restricted to the MTL (Knowlton et al., 1992; Knowlton \& Squire, 1994, 1996). Although the neuropathology of AD results in compromised functioning of the MTL, the progression of $\mathrm{AD}$ also involves damage to neocortical areas in the frontal lobe and additional temporal cortical regions (Braak \& Braak, 1991; Delacourte et al., 1999; Hyman et al., 1984; Kemper, 1984). The generally successful acquisition of the AGL by AD patients suggests that this neuropathology outside of the hippocampus and the entorhinal cortex did not strongly interfere with learning on this task. It should also be noted that the progression of $\mathrm{AD}$ can be somewhat heterogeneous with respect to the brain areas exhibiting neuropathology. In some cases, AD patients might have damage in areas supporting AGL, possibly reflected in the observed trend for poorer overall group performance.

The dissociation between performance on the grammaticality judgment and recognition tasks implies that there are separate memory systems for acquiring these types of memories. An important prerequisite for understanding the organization of memory across the brain is to assess the degree to which these memory systems interact or act independently. Here, the recognition memory test was given with a set of grammatical study items, a novel approach that potentially enabled the participants to use grammaticality to guide recognition decisions. During the recognition task, both the $\mathrm{AD}$ patients and the controls often endorsed new grammatical items as having been seen before, indicating that the grammaticality of the test strings influenced recognition judgments. Unlike the controls, however, the AD patients did not discriminate between the old and the new grammatical items in their recognition responses (particularly the most memoryimpaired patients). This implies that when declarative memory was impaired, the patients attempted to use their intact nondeclarative memory to perform the recognition test, suggesting that these types of memory can interact. 
In contrast to this interaction, previous studies in which the relationship between declarative and nondeclarative memory has been examined using priming and recognition tasks have reported a surprising degree of independence between the systems, suggesting that behavior tends to reflect the contribution of one system or the other. A series of studies of the densely amnesic patient E.P. has shown that his priming is normal, although he is consistently unable to perform recognition judgments at better than chance (Hamann \& Squire, 1997; Stark \& Squire, 2000), suggesting that the intact priming does not contribute to recognition judgments. Other studies with amnesic patients have also reported independent operation of priming and recognition (e.g., Knowlton \& Squire, 1995; P. J. Reber \& Squire, 1999b). In addition, recent functional neuroimaging studies (Donaldson, Petersen, \& Buckner, 2001) have suggested that separate brain systems are involved in priming and recognition (although these do not rule out the possibility that information from both systems is integrated at response time). However, these reports of independentoperation are not uncontroversial, since some reports have suggested an influence of priming on recognition (e.g., Johnston, Dark, \& Jacoby, 1985; Verfaellie \& Cermak, 1999).

The comparison between priming and AGL may be informative for understanding differences across types of implicit memory. If priming and recognition operate independently, whereas AGL influences recognition judgments, this suggests that different mechanisms support these two types of implicit learning. It is reasonable to hypothesize that these two tasks are supported by separate mechanisms, since perceptual priming tends to be tied to the perceptual characteristics of task stimuli (Biederman \& Cooper, 1991), whereas AGL appears to be able to support complex and highly abstract transfer. Participants have been shown to be able to make grammaticality decisions on stimuli for which all the surface perceptual characteristics have been changed - that is, when the constituent letters of the strings have all been replaced by novel letters for the test (Knowlton \& Squire, 1996; A. S. Reber, 1989). Although the present study did not examine the ability of AD patients to perform the letter-set transfer version of AGL, even a perceptual fragment-based learning hypothesis for AGL in AD implies an additional, more flexible nondeclarative memory ability in $\mathrm{AD}$ over the previous reports of intact perceptual priming.

Because the AD patients are successful at AGL, we suggest that this task is likely to be supported by more posterior cortical areas that are less affected early in AD. Functional neuroimaging of AGL has identified changes in activity in posterior, inferior parietal brain regions associated with this type of memory (Skosnik, Gitelman, Parrish, Mesulam, \& Reber, 2002). Although it might seem counterintuitive to suggest that this nominal "grammar" task relies on posterior (nonlanguage) areas, AGL requires identification of correct sequences of symbolic characters without semantic meaning and may not be supported by traditional language-processing areas of the brain. Our suggestion that posterior cortical areas support AGL is somewhat similar to the case of perceptual priming, yet it is clear that the AGL task demands a more complex and abstract representation of knowledge than does perceptual priming, indicating that a different mechanism is likely to be involved. The idea of separate mechanisms to support AGL and perceptual priming is further supported by the fact that AGL knowledge appears to influence recognition memory performance more readily than does priming.

\section{CONCLUSIONS}

Patients with early AD successfully learned the AGL task, generally performing similarly to a group of age- and education-matched controls. A trend for poorer performance by the AD patients suggests that mild AD may begin to affect implicit learning of artificial grammar rules. However, the neural substrate of this conceptual and cognitively complex nondeclarative memory function does not appear to be compromised by the most common anatomical distribution of neuropathology in early AD. Recognition memory, as was expected, was found to be impaired in the $\mathrm{AD}$ patients, reflecting their declarative memory dysfunction. In addition, the AD patients appeared to be able to use the grammaticality of study items to guide their responses on the recognition task, suggesting that for the AGL task, intact nondeclarative memory can influence declarative memory performance. These results reinforce the idea that nondeclarative memory consists of a collection of component processes, dependent on distinct brain areas, that differ in their processing capacities and interactions with declarative memory.

\section{REFERENCES}

Biederman, I., \& CoOper, E. E. (1991). Evidence for complete translational and reflectional invariance in visual object priming. Perception, 20, 585-593.

BRAAK, H., \& BRAAK, E. (1991). Neuropathological staging of Alzheimerrelated changes. Acta Neuropathologica, 82, 239-259.

Corkin, S. (1982). Some relationships between global amnesias and the memory impairments in Alzheimer's disease. In S. Corkin (Ed.), Alzheimer's disease: A report of progress (Aging, Vol. 19, pp. 149164). New York: Raven.

Delacourte, A., David, J. P., Sergeant, N., Buée, L., Wattez, A., Vermersch, P., Ghozali, F., Fallet-Bianco, C., Pasquier, F., Lebert, F., Petit, H., \& Di Menza, C. (1999). The biochemical pathway of neurofibrillary degeneration in aging and Alzheimer's disease. Neurology, 52, 1158-1165.

Donaldson, D. I., Petersen S. E., \& Buckner R. L. (2001). Dissociating memory retrieval processes using f MRI: Evidence that priming does not support recognition memory. Neuron, 31, 1047-1059.

Eslinger, P. J., \& DAmasio, A. R. (1986). Preserved motor learning in Alzheimer's disease: Implications for anatomy and behavior. Journal of Neuroscience, 6, 3006-3009.

Fleischman, D. A., \& Gabrieli, J. D. E. (1998). Repetition priming in normal aging and Alzheimer's disease: A review of findings and theories. Psychology \& Aging, 13, 88-119.

Folstein, M. F., Folstein, S. E., \& McHugh, P. R. (1975). Mini-mental state: A practical method for grading the cognitive state of patients for the clinician. Journal of Psychiatric Research, 12, 189-198.

Gabrieli, J. D. E., Vaidya, C. J., Stone, M., Francis, W. S., Thompson- 
Schill, S. L., Fleischman, D. A., Tinklenberg, J. R, Yesavage, J. A., \& WILSON, R. S. (1999). Convergent behavioral and neuropsychological evidence for a distinction between identification and production forms of repetition priming. Journal of Experimental Psychology: General, 128, 479-498.

Hamann, S. B., \& Squire, L. R. (1997). Intact perceptual memory in the absence of conscious memory. Behavioral Neuroscience, 111, 850-854.

Heindel, W. C., Salmon, D. P., \& Butters, N. (1990). Pictorial priming and cued recall in Alzhiemer's and Huntington's disease. Brain \& Cognition, 13, 282-295.

Heindel, W. C., Salmon, D. P. Shults, C. W., Walicke, P. A., \& ButTERS, N. (1989). Neuropsychological evidence for multiple implicit memory systems: A comparison of Alzheimer's, Huntington's, and Parkinson's disease patients. Journal of Neuroscience, 9, 582-587.

Hyman, B. T., Van Horsen, G. W., Damasio, A. R., \& Barnes, C. L. (1984). Alzheimer's disease: Cell-specific pathology isolates the hippocampal formation. Science, 225, 1168-1170.

Jackson, G. M., Jackson, S. R., Harrison, J., Henderson, L., \& KenNARD, C. (1995). Serial reaction time learning and Parkinson's disease: Evidence for a procedural learning deficit. Neuropsychologia, 33, 577-593.

Johnston, W. A., DARK, V. J., \& JACOBY, L. L. (1985). Perceptual fluency and recognition judgments. Journal of Experimental Psychology: Learning, Memory, \& Cognition, 11, 3-11.

Kaplan, E., Goodglass, H., \& Weintraub, S. (1983). The Boston Naming Test. Philadelphia: Lea and Febiger.

Keane, M. M., Gabrieli, J. D. E., Fennema, A. C., Growdon, J. H., \& CoRKIN, S. (1991). Evidence for a dissociation between perceptual and conceptual priming in Alzheimer's disease. Behavioral Neuroscience, 105, 326-342.

KemPER, T. (1984). Neuroanatomical and neuropathological changes in normal aging and dementia. In M. L. Albert (Ed.), Clinical neurology of aging (pp. 9-52). New York: Oxford University Press.

Kéri, S., Kálmán, J., Kelemen, O., Benedek, G., \& Janka, Z (2001). Are Alzheimer's disease patients able to learn visual prototypes? Neuropsychologia, 39, 1218-1223.

Kéri,. S., Kálmán, J., Rapcsak, S. Z, Antal, A., Benedek, G., \& JANKA, Z. (1999). Classification learning in Alzheimer's disease. Brain, 122, 1063-1068.

KNOPMAN, D. S., \& NisSEN, M. J. (1987). Implicit learning in patients with probable Alzheimer's disease. Neurology, 37, 784-788.

Knowlton, B. J., Mangels, J. A., \& Squire L. R. (1996). A neostriatal habit learning system in humans. Science, 273, 1399-1402.

Knowlton, B. J., Ramus, S. J., \& SQuire, L. R. (1992). Intact artificial grammar learning in amnesia: Dissociation of classification learning and explicit memory for specific instances. Psychological Science, 3, 172-179.

Knowlton, B. J., \& Squire, L. R. (1994). The information acquired during artificial grammar learning. Journal of Experimental Psychology: Learning, Memory, \& Cognition, 20, 79-91.

Knowlton, B. J., \& SQuire, L. R. (1995). Remembering and knowing: Two different expressions of declarative memory. Journal of Experimental Psychology: Learning, Memory, \& Cognition, 21, 699-710.

Knowlton, B. J., \& SQuire, L. R. (1996). Artificial grammar learning depends on implicit acquisition of both abstract and exemplar-specific information. Journal of Experiment Psychology: Learning, Memory, \& Cognition, 22, 169-181.

McKhann, G., Drachman, D., Folstein, M., Katzman, R., Price, D., \& Stadlan, E. M. (1984). Clinical diagnosis of Alzheimer's disease: Report of the NINCDS-ADRDA Work Group under the auspices of Department of Health and Human Services Task Force on Alzheimer's Disease. Neurology, 34, 939-949.

Monti, L. A., Gabrieli, J. D. E., Remlinger, S. L., Rinaldi, J. A., Wilson, R. S., \& Fleischman, D. A. (1996). Differential effects of aging and Alzheimer's disease on conceptual implicit and explicit memory. Neuropsychology, 10, 101-112.

Morris, J. C. (1993). The Clinical Dementia Rating (CDR): Current version and scoring rules. Neurology, 43, 2412-2414.
Morris, J. C., Heyman, A., Mohs, R. C., Hughes, J. P., van Belle, G., Fillenbaum, G., Mellits, E. D., \& Clark, C. (1989). The Consortium to Establish a Registry for Alzheimer's Disease (CERAD): Pt. I. Clinical and neuropsychological assessment of Alzheimer's disease. Neurology, 39, 1159-1165.

Nebes, R. D. (1989). Semantic memory in Alzheimer's disease. Psychological Bulletin, 106, 377-394.

Nebes, R. D. (1992). Cognitive dysfunction in Alzheimer's disease. In F. I. M. Craik \& T. A. Salthouse (Eds.), The handbook of aging and cognition (pp. 373-446). Hillsdale, NJ: Erlbaum.

Nissen, M. J., \& Bullemer, P. (1987). Attentional requirements of learning: Evidence from performance measures. Cognitive Psychology, 19, 1-32.

Palmeri, T. J., \& Flanery, M. A. (1999). Learning about categories in the absence of training: Profound amnesia and the relationship between perceptual categorization and recognition memory. Psychological Science, 10, 526-530.

Peigneux, P., Meulemans, T., Van Der Linden, M., Salmon, E., \& Petti, H. (1999). Exploration of implicit artificial grammar learning in Parkinson's disease. Acta Neurologica, 99, 107-117.

Perruchet, P. (1994). Defining the knowledge units of a synthetic language: Comment on Vokey and Brooks (1992). Journal of Experimental Psychology: Learning, Memory, \& Cognition, 20, 223-228.

REBER, A. S. (1967). Implicit learning of artificial grammars. Journal of Verbal Learning \& Verbal Behavior, 6, 855-863.

Reber, A. S. (1989). Implicit learning and tacit knowledge. Journal of Experimental Psychology: General, 118, 219-235.

Reber, P. J., \& SQuire, L. R. (1994). Parallel brain systems for learning with and without awareness. Learning \& Memory, 1, 217-229.

Reber, P. J., \& SQuire, L. R. (1999a). Intact learning of artificial grammars and intact category learning by patients with Parkinson's disease. Behavioral Neuroscience, 113, 235-242.

Reber, P. J., \& Squire, L. R. (1999b). Relaxing decision criteria does not improve recognition memory in amnesic patients. Memory \& Cognition, 27, 501-511

Reber, P. J., Stark, C. E. L., \& SQuire, L. R. (1998a). Contrasting cortical activity associated with declarative and nondeclarative memory. Learning \& Memory, 5, 420-428.

Reber, P. J., STARK, C. E. L., \& SQuire, L. R. (1998b). Cortical areas supporting category learning identified using functional magnetic resonance imaging. Proceedings of the National Academy of Sciences, 95, 747-750.

Rosler, A., Mapstone, M. E., Hays, A. K., Mesulam, M. M., Rademaker, A., Gitelman, D. R, \& Weintraub, S. (2000). Alterations of visual search strategy in Alzheimer's disease and aging. Neuropsychology, 14, 398-408.

Schacter, D. L., \& Buckner, R. L. (1998). Priming and the brain. Neuron, 20, 185-195.

Skosnik, P. D., Gitelman, D. R., Parrish, T. B., Mesulam, M. -M., \& REBER, P. J. (2002). Neural correlates of artificial grammar learning. NeuroImage, 17, 1306-1314.

SQuire, L. R., Knowlton, B. J., \& Musen, G. (1993). The structure and organization of memory. Annual Review of Psychology, 44, 453-495.

Stark, C. E. L., \& Squire, L. R. (2000). Recognition memory and familiarity judgments in severe amnesia: No evidence for a contribution of repetition priming. Behavioral Neuroscience, 114, 459-467.

Verfaellie, M., \& Cermak, L. S. (1999). Perceptual fluency as a cue for recognition judgments in amnesia. Neuropsychology, 13, 198-205.

Vokey, J. R., \& Brooks, L. R. (1992). Salience of item knowledge in learning artificial grammars. Journal of Experimental Psychology: Learning, Memory, \& Cognition, 18, 328-344.

Wagner, A. D., Koutstaal, W., Maril, A., Schacter, D. L., \& BuCKNER, R. L. (2000). Task-specific repetition priming in left inferior prefrontal cortex. Cerebral Cortex, 10, 1176-1184.

WeCHSLER, D. (1987). Wechsler Memory Scale-Revised manual. San Antonio, TX: The Psychological Corporation.

(Manuscript received June 29, 2001; revision accepted for publication March 22, 2003.) 